\title{
Checkpoint blockade in unresectable pleural mesothelioma: Event horizon for multimodal therapy
}

\author{
R. Taylor Ripley, MD, ${ }^{\mathrm{a}}$ Aaron S. Mansfield, MD, ${ }^{\mathrm{b}}$ Boris Sepesi, MD, ${ }^{\mathrm{c}}$ Raphael Bueno, MD, ${ }^{\mathrm{d}}$ and \\ Bryan M. Burt, MD
}

The landmark CheckMate 743 clinical trial of checkpoint blockade for malignant pleural mesothelioma (MPM) was published recently in the Lancet $^{1}$ and, in short order, has changed clinical practice. This multinational, multiinstitutional phase 3 trial randomized patients with untreated and unresectable MPM to dual-agent checkpoint blockade with anti-programmed cell death protein 1 (PD1) (nivolumab) plus anti-cytotoxic T-lymphocyte antigen [CTLA]-4 therapy (ipilimumab) or systemic chemotherapy with cisplatin plus pemetrexed. The output of this trial has resulted in United States Food and Drug Administration (FDA) approval of immunotherapy as front-line therapy for MPM, revision of National Comprehensive Care Network guidelines in support of this therapy in the first line, and thus, a momentous change in our standards of care. This Feature Expert Opinion Article is intended to review the salient results of this groundbreaking trial and to enhance its careful translation.

\section{COHORT}

Appropriate application of the results of CheckMate 743 to clinical practice begins with understanding eligibility for its enrollment. Patients in CheckMate 743 had histologically confirmed epithelioid, biphasic, or sarcomatoid MPM tumors; had no previous treatment; and were determined to be unresectable. Exclusion criteria included untreated brain metastases, nonpleural mesotheliomas, and recent corticosteroid or immunosuppressive medications. In balance, this cohort is generally representative of most patients encountered in the clinic who are newly diagnosed with MPM. However, performance status quantified as Eastern Cooperative Oncology Group 0 to 1 was required to enter

\footnotetext{
From the ${ }^{\mathrm{a} D i v i s i o n}$ of General Thoracic Surgery, Michael E. DeBakey Department of Surgery, Baylor College of Medicine, Houston, Tex; ${ }^{b}$ Division of Medical Oncology, Mayo Clinic, Rochester, Minn; ${ }^{\mathrm{c}}$ Department of Thoracic and Cardiovascular Surgery, University of Texas MD Anderson Cancer Center, Houston, Tex; and ${ }^{\mathrm{d}}$ Division of Thoracic Surgery, Brigham and Women's Hospital, Boston, Mass.

Received for publication June 24, 2021; revisions received Nov 27, 2021; accepted for publication Jan 11, 2022; available ahead of print Jan 22, 2022.

Address for reprints: Bryan M. Burt, MD, Division of General Thoracic Surgery, Department of Surgery, Baylor College of Medicine, One Baylor Plaza, MS BCM 390, Houston, TX 77030 (E-mail: bryan.burt@bcm.edu).

J Thorac Cardiovasc Surg 2023;165:364-8

0022-5223/\$36.00

Copyright (C) 2022 by The American Association for Thoracic Surgery

https://doi.org/10.1016/j.jtcvs.2022.01.020
}

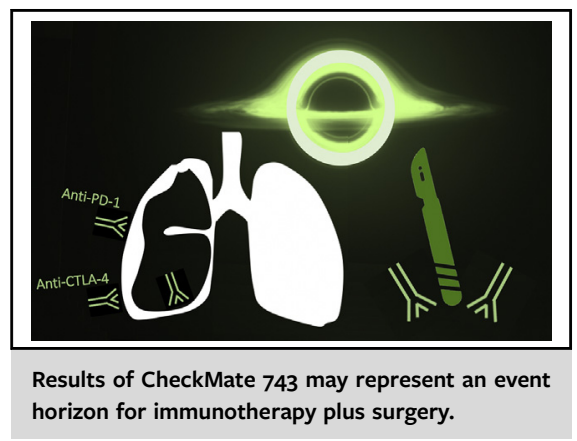

CENTRAL MESSAGE

Dual-agent checkpoint blockade significantly improved survival in patients with unresectable malignant pleural mesothelioma in the CheckMate 743 trial.

This Invited Expert Opinion provides a perspec tive on the following paper: Lancet. 2021 Jan 30;397(10272):375-386 So140-6736(20)32714-8. Epub 2021 Jan 21

See Commentary on page 369.

this trial, and clinical practice encounters with patients with MPM who are less fit are quite common. Importantly, CheckMate 743 included only patients with unresectable disease, and resectability was determined by the investigator at individual sites using local standards. These locally based criteria are important because determination of operable disease in MPM is notoriously nuanced, can include potentially biased impressions of fitness for surgery ("medical unresectability"), and ultimately defines a crossroads for selecting appropriate therapy. A reasonable suggestion for future MPM clinical trial design is a requirement for surgeon input to estimate the likelihood of safe and successful macroscopic complete resection. ${ }^{2}$

\section{REGIMEN}

In patients with unresectable previously treated MPM, single-agent immune checkpoint blockade therapy against PD-1 or PD-L1 has demonstrated objective response rates of $8 \%$ to $29 \%$ and disease control in $40 \%$ to $52 \%$ of 
patients. $^{3}$ A large, multicenter randomized trial, antiCTLA-4 monotherapy with tremelimumab, did not improve overall survival (OS) compared with placebo in previously treated patients. ${ }^{4}$ Combining blockade of both PD-1/PD-L1 and CTLA-4 checkpoints is being investigated in a number of human malignancies to improve immunologic responses.

The specific immunotherapeutic regimen tested in CheckMate 743 is important to review, as it will likely become a benchmark against which next-generation strategies will be tested. Patients were randomized 1:1 to the PD1 inhibitor nivolumab (3 mg/kg intravenous infusion once every 2 weeks) plus the CTLA-4 inhibitor ipilimumab ( $1 \mathrm{mg} / \mathrm{kg}$ intravenous infusion once every 6 weeks), or platinum-based chemotherapy (cisplatin $\left[75 \mathrm{mg} / \mathrm{m}^{2}\right]$ or carboplatin [area under the time-concentration curve $5 \mathrm{mg} / \mathrm{mL} /$ $\mathrm{min}]$ ) plus pemetrexed $\left(500 \mathrm{mg} / \mathrm{m}^{2}\right)$. Patients were stratified by sex and histology (epithelioid vs nonepithelioid), and $75 \%$ of patients had epithelioid MPM. Treatment assignment was not blinded to patients or investigators after randomization, and treatment was continued until progression, until unacceptable toxicity, or for 2 years. Ultimately approved by the FDA was a regimen of $360 \mathrm{mg}$ of nivolumab every third week.

A thorough understanding of the chemotherapy regimen of the control group is just as important to contextualize the benefits of the immunotherapy regimen within the current treatment landscape. When published in 2003, cisplatin plus pemetrexed chemotherapy showed a survival extension of 2.8 months over cisplatin alone (12.1 vs 9.3 months). ${ }^{5}$ Although the median survival of 18.1 months demonstrated with dual-agent checkpoint blockade in CheckMate 743 is a meaningful improvement over the 12.1 months in the chemotherapy doublet trial, we must also consider the results of the more contemporary Mesothelioma Avastin Cisplatin Pemetrexed (MAPS) Trial, published in 2016. In MAPS, 448 patients with unresectable MPM were randomized to platinum plus pemetrexed with or without the antiangiogenic vascular endothelial growth factor inhibitor, bevacizumab. The addition of bevacizumab improved median survival over chemotherapy alone (18.8 vs 16.1 months $)^{6}$; however, the combination of bevacizumab plus chemotherapy is not currently approved by the FDA. CheckMate 743 did not incorporate bevacizumab regimens, and how dual-agent checkpoint blockade measures up to a chemotherapy triplet with bevacizumab is unknown. However, the outcomes of the immunotherapy arm in CheckMate 743 and the bevacizumab arm in MAPS are not dissimilar.

\section{ENDPOINT}

The CheckMate 743 trial was designed to test a primary end point of OS, and power calculations estimated that 600 patients with 473 deaths would provide $90 \%$ power to detect a hazard ratio (HR) of 0.72 with a 2 -sided type I error of 0.05 . A prespecified interim analysis was planned at 403 deaths to assess for superiority. Among the 713 patients who were enrolled, 605 were randomly assigned to checkpoint blockade $(\mathrm{n}=303)$ or chemotherapy $(\mathrm{n}=302)$ and patient characteristics including sex, histology, and tumor PD-L1 expression were well balanced between the groups. A prespecified interim analysis was performed after 419 deaths, at a median follow-up of 29.7 months. At this time point, a significant benefit in median OS was demonstrated for dual-agent checkpoint blockade compared with chemotherapy (18.1 vs 14.1 months, respectively). At 1 year, rates of OS were $68 \%$ and $58 \%$ for dual-agent checkpoint blockade and chemotherapy, respectively, and at 2 years were $41 \%$ and $27 \%$. These data are overall supportive of the efficacy of dual agent immune checkpoint blockade in MPM patients with good performance status.

\section{SUBGROUPS}

The histology of MPM is important to consider in any study that evaluates clinical outcomes. Patients with nonepithelioid tumor histology (sarcomatoid or biphasic) have traditionally demonstrated worse outcomes than patients with epithelioid tumor histology in essentially all treatment settings, until now. Generally representative of the epidemiology of MPM, $25 \%$ of patients participating in CheckMate 743 had nonepithelioid tumors. Although OS was improved with nivolumab and ipilimumab compared with chemotherapy regardless of tumor histology, compelling evidence was presented for a greater treatment effect in patients with nonepithelioid histology. Patients with epithelioid MPM who were treated with checkpoint inhibition had a median OS of 18.7 compared with 16.5 months in those treated with chemotherapy (HR, 0.86 [0.69-1.08]). In contrast, patients with nonepithelioid MPM treated with checkpoint inhibition had markedly different median OS than those treated with chemotherapy, 18.1 versus 8.8 months, respectively (HR, 0.46 [0.31-0.68]). Interestingly, patients with nonepithelioid MPM who received checkpoint inhibitors had nearly the same survival outcomes as the patients with epithelioid histology MPM. In other words, the improvement over chemotherapy was more significant the nonepithelioid group compared with the epithelioid group and is likely related to the differential responsiveness to chemotherapy between epithelioid and nonepithelioid MPM.

\section{TOXICITY}

A valid concern in evaluating checkpoint blockade regimens, particularly dual-agent strategies, is toxicity. Despite the improvement in outcomes in Checkmate 743, immunerelated adverse events were not infrequent, and patients must be counseled on the toxicity profile of this combination. In general, there were similar frequencies of any grade 1 to $2(49 \%$ vs $50 \%)$, grade $3(26 \%$ vs $26 \%)$ and grade 4 
(4\% vs $6 \%)$ events between the immunotherapy and chemotherapy treatment arms, respectively. Diarrhea likely due to immune-related colitis was one of the most frequent side effects with the immunotherapy arm, whereas anemia was more common in the chemotherapy arm. Adverse events led to discontinuation of treatment in a substantial $23 \%$ of patients receiving immunotherapy, compared with $16 \%$ of patients who those administered chemotherapy. Granted, the planned course of chemotherapy was shorter than the planned immunotherapy regimen.

\section{BIOMARKERS}

There is currently no reliable biomarker to accurately identify MPM patients who will respond to immune checkpoint blockade. Such a test would avoid unnecessary toxicity, triage nonresponders to potentially more effective treatment, and could even extend long-term survival. In CheckMate 743, tumor PD-L1 expression was explored as a predictive biomarker; however, interpretation of these results was cautioned based on lack of stratification by PD-L1 expression and by a small sample size in the PD-L1 low group. The investigators demonstrated that OS outcomes with nivolumab plus ipilimumab were similar in the subgroups with $<1 \%$ and with $\geq 1 \%$ tumor PD-L1 expression, with better outcomes seen with nivolumab plus ipilimumab than with chemotherapy at 2 years in both subgroups. Whereas high tumor mutation burden and neoantigen burden have associated with response to checkpoint inhibition in other carcinogen-driven tumors such as lung cancer and melanoma, MPM has a surprisingly low number of mutations, even though it is driven by the asbestos carcinogen. We anticipate that ongoing translational investigation will reveal novel genomic and proteomic biomarkers for predicting and monitoring response to immune checkpoint blockade in MPM.

\section{PRESENT}

Based on the results of Checkmate-743, the FDA approved nivolumab plus ipilimumab for patients with unresectable MPM of any histology in October of 2020. This approval has significant implications for the current state of the field. First, more frontline options are now available for medical oncology teams, who can now select between combination nivolumab and ipilimumab or platinum-based therapy with pemetrexed, with or without bevacizumab. Second, for patients with sarcomatoid and biphasic MPM, this approval has ushered into the first line a therapy with remarkable effectiveness compared with existing therapies. Third, the establishment of checkpoint blockade into first-line systemic therapies for patients with MPM will facilitate future innovative trial design that could further improve outcomes for patients suffering from MPM.

\section{FUTURE}

Whereas data from the CheckMate 743 trial are very exciting, the results of the state-of-the-art systemic therapy for patients with MPM are still sobering: complete responses occurred in $2 \%$ of patients treated with checkpoint inhibitors and $0 \%$ in patients treated with chemotherapy. This challenge is familiar to the field and one that has been traditionally approached by combining multiple therapeutic modalities.

\section{Chemoimmunotherapy}

Chemoimmunotherapy is a natural progression of investigations in line with this spirit and was tested in the recently completed phase 2 PrE0505 multicenter trial that demonstrated that adding durvalumab (a PD-L1 checkpoint inhibitor) to cisplatin and pemetrexed chemotherapy improved outcomes in previously untreated, unresectable patients. The median OS of chemoimmunotherapy treated patients was of 20.4 months compared with 12.1 months for historical controls. OS rates at 12 and 24 months for those treated with chemoimmunotherapy were $70.4 \%$ and $44.2 \%$, respectively. ${ }^{7}$ As mentioned previously, historical control data of 12.1-month median survival may underestimate current expected outcomes, but 20.4 months is one of the highest reported median OS to date. Immunogenomic analyses demonstrated that a greater immunogenic mutation burden coupled with a diverse T-cell repertoire correlated with favorable clinical outcome.

\section{Neoadjuvant Immunotherapy}

At the forefront of checkpoint blockade for MPM is the neoadjuvant setting. MPM is a unique tumor with a dense, fibrotic, and circulation-deplete stroma that limits drug delivery, and large databases studies have supported a role for surgical resection in improving survival when adopted into multimodality therapy that commonly includes systemic therapy. ${ }^{8,9}$ There are a number of theoretical advantages of neoadjuvant checkpoint blockade, including initiation of a memory immune response that might be recalled after the bulk of the tumor is removed and which could be bolstered by additional immunotherapy in the adjuvant setting. Other hypothetical advantages include improvement of technical resectability and/or disease downstaging. The results of several relevant contemporary trials will inform the field on the safety, feasibility, and efficacy of this approach (Table 1).

Directly relevant to the checkpoint blockade regimen used in CheckMate 743, NCT03918252 is recruiting patients with MPM to receive nivolumab plus ipilimumab in the neoadjuvant setting and is designed to test primary end points of safety and feasibility. This trial is open to patients with epithelioid or biphasic MPM and is investigating secondary objectives including pathologic and radiographic 
TABLE 1. Neoadjuvant immune checkpoint blockade trials in MPM

\begin{tabular}{|c|c|c|c|c|c|}
\hline NCT & Phase & Neoadjuvant therapy & Sample size & Primary end point & Status \\
\hline NCT02592551 & II & Durvalumab vs durvalumab plus tremelimumab & 16 & Intratumoral $\mathrm{CD} 8 \mathrm{~T}$ cell/Treg ratio & Closed to accrual \\
\hline NCT02707666 & I & Pembrolizumab & 15 & $\begin{array}{l}\gamma \text {-Interferon gene expression } \\
\text { profile and safety }\end{array}$ & Accruing \\
\hline NCT03918252 & I-II & Nivolumab vs nivolumab plus ipilimumab & 30 & Safety and feasibility & Accruing \\
\hline NCT04162015 & I & Nivolumab plus platinum/pemetrexed & 35 & Feasibility & Accruing \\
\hline NCT03228537 & I & Cisplatin/pemetrexed plus atezolizumab & 24 & Feasibility and safety & Closed to accrual \\
\hline
\end{tabular}

Durvalumab: anti-PD-L1; tremelimumab: anti-CTLA-4; pembrolizumab: anti-PD-1; nivolumab: anti-PD-1; ipilimumab: anti-CTLA-4. NCT, National clinical trial number; Treg, Regulatory T cells.

response. In a pilot phase I study of the anti-PD-1 antibody pembrolizumab, investigators are determining the change in interferon-gamma gene signature, and are assessing safety in patients with epithelial or biphasic MPM (NCT02707666). Finally, we completed a neoadjuvant window-of-opportunity trial performed at Baylor College of Medicine (NCT02592551) in which we randomized patients with MPM to a single cycle of neoadjuvant durvalumab (anti-PD-L1) or durvalumab plus tremelimumab (anti-CTLA-4), and its early results were presented at the 2021 American Association for Thoracic Surgery annual meeting. ${ }^{10}$ The primary objectives of this study are biomarker-derived; however, a strong OS signal was observed in patients receiving dual-agent therapy compared with those receiving single-agent therapy $(15 \%$ vs $75 \%$ 2-year OS; median survival 14 months vs not reached).

\section{Neoadjuvant Chemoimmunotherapy}

Neoadjuvant chemoimmunotherapy was tested in Southwest Oncology Group 1619 (NCT03228537), which is now closed for accrual. This phase 1 study was designed to determine the safety and feasibility of the PDL1 inhibitor atezolizumab in combination with cisplatin and pemetrexed (with or without adjuvant radiotherapy) for patients with MPM. Survival metrics and changes in the tumor immune contexture are being studied as secondary objectives. In a single-institution pilot study of nivolumab combined with pemetrexed and platinum chemotherapy, investigators are investigating feasibility by determining the number of patients who arrive at the operating room for resection of any histology MPM (NCT04162015). Published data from each of these neoadjuvant immunotherapy and neoadjuvant chemoimmunotherapy trials are anticipated to support approaches incorporating surgery and checkpoint blockade for patients suffering from MPM.

\section{CONCLUSIONS}

Combined checkpoint inhibition with nivolumab and ipilimumab is one of the most significant advances in systemic therapy for MPM. In the practice-changing CheckMate 743 trial, the survival benefit of dual-agent checkpoint blockade was particularly remarkable for patients with nonepithelioid histology MPM. For patients with epithelioid MPM, nivolumab plus ipilimumab provides another first-line option; however, its comparative efficacy with chemotherapy that includes bevacizumab remains to be determined. Finally, integrating checkpoint blockade and surgical resection could potentially improve survival in patients with resectable epithelioid and nonepithelioid MPM.

\section{Conflict of Interest Statement}

R.T.R. reports research support from the National Institutes of Health and the American Association for Thoracic Surgery; nonremunerated board of director of the Mesothelioma Applied Research Foundation; and was retained to provide expert opinion. A.S.M. reports research support from Novartis and Verily; remuneration to his institution for participation on advisory boards for AbbVie, AstraZeneca, Bristol Myers Squibb, Genentech, and Janssen; travel support and payment from Shanghai Roche Pharmaceuticals Ltd; and is a nonremunerated director of the Mesothelioma Applied Research Foundation. B.S. has received consultant fees from Bristol Myers Squibb and speaker fees from AstraZeneca. R.B. reports research funding from the National Institutes of Health, Intuitive Surgical Foundation, Roche, Genentech, Merck, Siemens, Verastem, Gritstone, North-Pond, and Epizyme; has served as a consultant to Regeneron and on the scientific advisory board of Novocure, has equity interests in Navigation Sciences, and intellectual property interests through Brigham and Women's Hospital. B.M.B. reports research funding from the National Institutes of Health, Cancer Prevention Research Institute of Texas, and Momotaro Gene; clinical trial funding from AstraZeneca, Novartis, and Momotaro Gene; has been a consultant for AstraZeneca, is a proctor for Intuitive Surgical, and has intellectual property and equity interests in a start-up surgical device company, Bayou Surgical.

The Journal policy requires editors and reviewers to disclose conflicts of interest and to decline handling or reviewing manuscripts for which they may have a conflict of interest. The editors and reviewers of this article have no conflicts of interest. 


\section{References}

1. Baas P, Scherpereel A, Nowak AK, Fujimoto N, Peters S, Tsao AS, et al. Firstline nivolumab plus ipilimumab in unresectable malignant pleural mesothelioma (CheckMate 743): a multicentre, randomised, open-label, phase 3 trial. Lancet. 2021;397:375-86.

2. Rice D, Rusch V, Pass H, Asamura H, Nakano T, Edwards J, et al. Recommendations for uniform definitions of surgical techniques for malignant pleural mesothelioma: a consensus report of the International Association for the Study of Lung Cancer International Staging Committee and the International Mesothelioma Interest Group. International Association for the Study of Lung Cancer International Staging Committee and the International Mesothelioma Interest Group. J Thorac Oncol. 2011;6:1304-12.

3. Harber J, Kamata T, Pritchard C, Fennell D. Matter of TIME: the tumor-immune microenvironment of mesothelioma and implications for checkpoint blockade efficacy. J Immunother Cancer. 2021;9:e003032.

4. Maio M, Scherpereel A, Calabrò L, Aerts J, Perez SC, Bearz A, et al. Tremelimumab as second-line or third-line treatment in relapsed malignant mesothelioma (DETERMINE): a multicentre, international, randomised, double-blind, placebo-controlled phase 2b trial. Lancet Oncol. 2017;18: 1261-73.

5. Vogelzang NJ, Rusthoven JJ, Symanowski J, Denham C, Kaukel E, Ruffie P, et al. Phase III study of pemetrexed in combination with cisplatin versus cisplatin alone in patients with malignant pleural mesothelioma. J Clin Oncol. 2003;21: 2636-44.

6. Zalcman G, Mazieres J, Margery J, Greillier L, Audigier-Valette C, MoroSibilot D, et al. Bevacizumab for newly diagnosed pleural mesothelioma in the Mesothelioma Avastin Cisplatin Pemetrexed Study (MAPS): a randomised, controlled, open-label, phase 3 trial. Lancet. 2016;387:1405-14.

7. Forde PM, Anagnostou V, Sun Z, Dahlberg SE, Kindler HL, Niknafs N, et al. Durvalumab with platinum-pemetrexed for unresectable pleural mesothelioma: survival, genomic and immunologic analyses from the phase 2 PrE0505 trial. Nat Med. 2021;27:1910-20.

8. Nelson DB, Rice DC, Niu J, Atay S, Vaporciyan AA, Antonoff M, et al. Longterm survival outcomes of cancer-directed surgery for malignant pleural mesothelioma: propensity score matching analysis. J Clin Oncol. 2017;35: 3354-62.

9. Rusch VW, Giroux D, Kennedy C, Ruffini E, Cangir AK, Rice D, et al. Initial analysis of the international association for the study of lung cancer mesothelioma database. J Thorac Oncol. 2012;7:1631-9.

10. Burt BM, R M, Jang HJ, Ramos D, Splawn T, Almarez M, et al. A randomized trial of neoadjuvant PD-L1 versus PD-L1 Plus CTLA-4. Blockade in patients with malignant pleural mesothelioma. J Thorac Cardiovasc Surg. 2021 [Epub ahead of print].

Key Words: mesothelioma, pleura, checkpoint blockade, immunotherapy, checkpoint inhibitor, nivolumab, ipilimumab 